\title{
Cyclin D1 expression and its correlation with clinicopathological variables in colorectal carcinoma
}

\author{
Neha Sharma ${ }^{1}$, Taranveer Singh ${ }^{1}$, Arshdeep Kaur ${ }^{1}$, Mridu Manjari ${ }^{1}$, Shazia \\ Bashir $^{1}$ \\ ${ }^{I}$ Department of Pathology; Sri Guru Ram Das Institute of Medical Sciences \& Research, Punjab, India
}

\author{
Keywords: \\ Adenocarcinoma; \\ Colorectal carcinoma; \\ Cyclin D1;
}

\begin{abstract}
Background: This study was conducted to evaluate the expression of Cyclin D1 in colorectal carcinoma, and to correlate Cyclin D1 positivity with the grade of the tumor along with other parameters such as age, tumor location, lymph node metastasis, and vessel invasion.
\end{abstract}

Materials and Methods: The present cross-sectional study was conducted on 40 proven cases of colorectal carcinoma from October 2015 to October 2017. The tissues were fixed, processed, and stained with hematoxylin and eosin followed by immunohistochemical staining with Cyclin D1 using monoclonal antibodies.

Results: $75 \%$ of the cases were conventional adenocarcinoma while the rest were mucinous adenocarcinoma. Of the total colorectal carcinomas, $12.5 \%$ were Grade I, 50\% were Grade II and 37.5\% cases were Grade III. Cyclin D1 positivity was seen in $60 \%$ of cases with the most positivity in grade II tumors. A statistically significant correlation of Cyclin D1 was seen with age group (5th- 6th decade) and histological type of tumor. No significant correlation was seen of Cyclin D1 with lymph node involvement, depth of invasion, vascular invasion, or perineural invasion. A significant statistical correlation was seen with Crohn's-like reaction and peritumoral lymphocytic response.

Conclusions: Thus, it was concluded that a highly statistically significant correlation was seen with age group (5th- 6th decade) and histological type of tumor. No significant correlation was seen with Cyclin D1 expression with lymph node involvement, depth of invasion, vascular invasion, or perineural invasion. A significant statistical correlation was seen with Crohn's-like reaction and peritumoral lymphocytic response.

\section{Correspondence:}

Dr. Neha Sharma, $M D$

Assistant Professor, Department of Pathology

Sri Guru Ram Das Institute of Medical Sciences \& Research,

Amritsar, Punjab, India

ORCID ID: 0000-0002-5434-3645

Email:nehavikasaagu@gmail.com

Received : June $5^{\text {th }} 2020$; Accepted : March $23^{\text {rd }} 2021$

Citation: Sharma N, Singh T, Kaur A, Manjari M, Bashir S. Cyclin D1 expression and its correlation with clinicopathological variables in colorectal carcinoma. J Pathol Nep 2021;11:1868-72. DOI: 10.3126/jpn.v11i1.29291

Copyright: This is an open-access article distributed under the terms of the Creative Commons Attribution 4.0 International License, which permits unrestricted use, distribution, and reproduction in any medium, provided the original author and source are credited.

\section{INTRODUCTION}

Colorectal cancer (CRC) is a lethal disease of the large intestine leading to significant morbidity and mortality. ${ }^{1}$ The incidence of CRC worldwide is higher in men than in women and increases with age for both genders. It remains a public health problem accounting for it being the third most common cancer in men $(746,000$ cases, $10 \%$ of the total) and the second-most in women (614,000 cases, 9.2\% of the total) worldwide. ${ }^{2}$ Current literature suggests that over 86 percent of those diagnosed under the age of 50 are symptomatic at the time of diagnosis and this is associated with more advanced stages at diagnosis and poorer 
outcomes. ${ }^{3}$ Incidence of CRC increases with age and is rare before the age of 40 years.

The age-standardized incidence of CRC varies greatly in different regions of the world. This is emphasized from the epidemiological data records showing high incidence rates of 25.3 per 100,000 in Eastern Europe to 45.8 per 100,000 in Australia, with quite low incidence rates in India of about 2 to 8 per $100,000 .^{4}$

Most CRC are located in the sigmoid colon and rectum but there is evidence of changing distribution in recent years with an increasing proportion of more proximal carcinomas. ${ }^{5}$ Histological types of CRC are mainly adenocarcinoma, other tumors include leiomyosarcoma, lymphoma, melanoma, and neuroendocrine tumors. Adenocarcinoma accounts for about $90-95 \%$ of all CRC.

The single most important prognostic indicator of CRC is the extent of the tumor at the time of the diagnosis, the socalled stage. The Tumor Node Metastases (TNM) staging system of the American Joint Committee on Cancer/ Union for International Cancer Control is the preferred staging system for CRC. ${ }^{6}$ Five-year survival rate for colon cancer is $95 \%$ for tumors that have not penetrated the muscularis propria, $80 \%$ for those that have penetrated through muscularis propria but have not spread to lymph node and $20-40 \%$ for those that have metastasized.

Immunohistochemistry and molecular markers such as CEA (carcinoembryonic antigen), CA 19-9, MUC1 and MUC3, p53, cytokeratin, HER-2/neu, CDX2, PLAP, Cathepsin, $\mathrm{hCG}$, and Cyclin D1are performed as it is helpful in not only diagnosing but also predicting the prognosis and treatment.$^{7-9}$

Cyclin D1 overexpression has been shown to correlate with early cancer onset and tumor progression in various neoplasms. Cyclin D1 (a member of the cyclin protein family) is a nuclear marker. The gene encoding Cyclin D1 is located on chromosome 11q13 which is commonly amplified in many human cancers including colorectal cancer. The synthesis of Cyclin D1 is initiated during G1 and drives the G1/S phase transition. Cyclin D1 is one of the major cyclins produced. It interacts with four cyclindependent kinases (CDKs): CDK 2, 4,5, and 6. Cyclins, the regulatory subunits of CDKs, control the passage of proliferating cells through key checkpoints in the cell cycle. The deregulation of cyclin D1 expression results in loss of control of normal growth. This study was conducted to evaluate the expression of Cyclin D1 in colorectal carcinoma, and to correlate Cyclin D1 positivity with the grade of the tumor along with other parameters such as age, tumor location, lymph node metastasis, and vessel invasion.

\section{MATERIAL AND METHODS}

This cross-sectional study was conducted on 40 cases of colorectal cancer received in the Department of Pathology, Sri Guru Ram Das Institute of Medical Sciences and Research, Amritsar from October 2015 to October 2017. The tissues were formalin-fixed and paraffin-embedded and then stained for hematoxylin and eosin for histopathological typing and grading.

All the cases were then subjected to immunohistochemistry for Cyclin D1 expression. Positive and negative controls were run with every batch of the IHC. Brown nuclei were taken as positive for Cyclin D1. Depending on the intensity of staining, a score of 0 (no staining), 1(mild staining), 2(moderate staining), 3 (strong staining) was given to the cells. The percentage of positive cells was given proportion score $0(<5 \%), 1(5-25 \%), 2(26-50 \%), 3(51-75 \%)$ and 4 $(>75 \%)$.

Total Score (0-7) was calculated by adding Proportion Score and Intensity score (0-7). Cases with a total score of 0 were given negative expression, a total score of 1- 4 were given weak expression and strong expression was given to a total score of 5-7.

\section{RESULTS}

Out of the total 40 cases, females outnumbered males with $\mathrm{M}$ : $\mathrm{F}$ ratio of 2:3 with a maximum number of cases $(55 \%)$ seen in the $5^{\text {th }}$ and $6^{\text {th }}$ decade of life. The youngest patient was 28 -years and the oldest was 80 years of age. Major complaints encountered in the patients studied were abdominal pain, bleeding per rectum, and weight loss that presented mostly in a combination of one or the other. Of the total 40 cases, 21 were colectomies and 19 were biopsies. Radiologically majority of the tumors, $28 / 40$ cases $(70 \%)$ were located in the distal part of the colon (rectum and sigmoid colon), the next common being descending colon with the least common in the transverse colon. Marked variation in tumor size was observed with the majority of cases having tumor size between $2-5 \mathrm{~cm}(62 \%)$. No case was found with tumor size $<2.0 \mathrm{~cm}$.

Amidst 21 cases of colectomies, $62 \%$ had an infiltrative type of growth pattern. The profile of CRC comprised a maximum number of cases of conventional adenocarcinoma $(75 \%)$ with the remaining being mucinous adenocarcinoma (25\%).

All the 40 cases were graded into well-differentiated (Grade I), moderately differentiated (Grade II), and poorly differentiated (Grade III) adenocarcinomas, of which 5 cases were Grade I, 20 cases were Grade II and 15 cases fell into the category of Grade III. All the cases of mucinous adenocarcinoma as by convention were graded into poorly differentiated carcinomas or grade III. All the 21 resected specimens were examined for the depth of invasion by 


\begin{tabular}{lc} 
Table 1: Percentage of cells positive for Cyclin D1 \\
\hline Percentage of positive cells & No of Cases (\%) \\
\hline $\mathbf{5} \%$ & $2(8.3)$ \\
$\mathbf{5 - 2 5 \%}$ & $5(20.8)$ \\
\hline $\mathbf{2 6 - 5 0 \%}$ & $12(50.0)$ \\
\hline $\mathbf{5 1 - 7 5 \%}$ & $5(20.8)$ \\
\hline Total & $\mathbf{2 4}(\mathbf{1 0 0})$ \\
\hline
\end{tabular}

tumor cells. In 17 cases (81\%) tumor cells were seen invading the serosa while in 4 cases $(19 \%)$ it was seen infiltrating the muscle only. Only 3 cases $(14.3 \%)$ of the 21 showed perineural invasion while the vascular invasion was seen in 10 cases $(47.6 \%)$ only. Crohn's like Reaction (CLR) was seen in 7 cases $(17.5 \%)$ while peritumoral lymphocytic response (PTLR) was identified in 26 cases (65\%). Intra tumoural lymphocytic Response (ITLR) was seen in 14 cases $(35 \%)$. Lymph nodes were recovered in all the 21 resected specimens and of these 10 cases (47.7\%) showed secondary carcinomatous deposits.

IHC was performed on all 40 cases of CRC. The results were interpreted according to intensity of staining and percentage cell positivity, and then scoring was done.

Of all the 40 cases of CRC subjected to Cyclin D1 expression, 24 cases (60\%) showed positivity (Table1). In the majority of cases 20/24 (83.3\%) Cyclin D1 showed only nuclear staining while in only 4 cases $(16.7 \%)$ both nuclear and cytoplasmic staining pattern was noted. Percentage of cells varied from $<5 \%$ to $75 \%$ in 24 cases positive for Cyclin D1.

The intensity of staining varied from moderate to strong with 4 cases showing weak intensity, 8 moderate, and 12 strong intensity. When scoring was done, of the 24 positive cases 13 cases showed weak positivity while 11 showed strong positivity.

There was no significant correlation of Cyclin D1 expression with the sex of the patient (p-value: 0.073). In our study, it was found that the patients in the age group of 41-60 showed a higher positivity (77.3\%) with Cyclin D1 expression (p-value: 0.033 ). It was observed that Cyclin D1 positivity was more in tumors of descending colon and rectum, 68\% being positive as compared to other sites which were only $48 \%$ but no significant correlation was seen (p-value:
0.432). There was no statistical significance between Cyclin D1 expression and the size of the tumor (p-value: 0.697). Of the 30 conventional adenocarcinoma cases, $21(70 \%)$ were positive for Cyclin D1 expression whereas of the 10 mucinous adenocarcinoma cases $3(30 \%)$ showed Cyclin D1 positivity. Thus, giving a significant correlation between the histological type of tumor and Cyclin D1 expression with a p-value of 0.025 . Correlation of Cyclin D1 expression was done with histological grade of the tumor. Of the 5-grade 1 tumor cases $2(40 \%)$ showed Cyclin D1 positivity, 15 of $20(75 \%)$ cases of grade II, and 7 cases of 15 grade III (46.6\%) were positive for Cyclin D1 expression. Grade III included all the cases of mucinous adenocarcinoma as per conventions of grading by WHO. Although the percentage of positivity increased from grade I to Grade II, it decreased as the grade further deteriorated from Grade II to Grade III. Thus, no statistically significant correlation of Cyclin D1 expression and grade of tumor was noted.

In all the 21 resected cases depth of invasion of tumor cells, perineural and vascular invasion of tumor cells were looked for and reported accordingly and their correlation with Cyclin D1 expression was checked. It was seen that none of these three parameters had any significant correlation with Cyclin D1 expression as was seen by their $\mathrm{p}$ values which were $0.149,0.368$, and 0.528 respectively. All these parameters were evaluated to find out the patient's immune response to the tumor and it was observed that Cyclin D1 expression was $100 \%$ in cases of CLR, $76.7 \%$ of PTLR, and $57.1 \%$ of ITLR (Table 2). The p-value of 0.017 and 0.003 had a highly significant correlation with CLR and PTLR respectively but no such significance was seen with ITLR having a p-value of 0.787 .

Ten cases of the 21 colectomies studied showed lymph node metastasis and 6 cases $(60 \%)$ among these showed Cyclin D1 expression while of the 10 cases where no metastasis was found, 6 cases (54.5\%) showed expression of Cyclin D1. There was no significant correlation between lymph node status and Cyclin D1 expression (p-value: 0.801).

Thus, it was observed that Cyclin D1 positivity correlated with age of the patient, histological type of adenocarcinoma, Crohn's like reaction, and peritumoral lymphocytic response but no definite significance was seen when correlated with sex, lymph node status, morphological distribution of tumor, size of tumor or grade of the tumor.

Table 2: Correlation of Cyclin D1 expression with Crohn's like reaction (CLR), peritumoral lymphocytic response (PTLR), and intratumoral lymphocytic response (ITLR)

\begin{tabular}{ccccccccc}
\hline & & & CLR & & \multicolumn{2}{c}{ PTLR } & \multicolumn{2}{c}{ ITLR } \\
& & Present & Absent & Present & Absent & Present & Absent \\
\multirow{2}{*}{ Cyclin D1 } & Positive & 7 & 17 & 20 & 4 & 8 & 16 \\
\hline & Negative & 0 & 16 & 06 & 10 & 6 & 10 \\
\hline & Percentage & 100 & 51.5 & 76.9 & 40 & 57.1 & 61.5 \\
\hline
\end{tabular}

The p-Value for CLR, PTLR, ITLR was 0.017(Significant), 0.003(Significant), and 0.787 (Not Significant) respectively.; Chi-square test 


\section{DISCUSSION}

In the present study, IHC staining for Cyclin D1 was performed in all 40 cases of CRC, and scoring as well as results were interpreted in terms of percentage of cell positivity, and staining intensity. Immunohistochemical expression of Cyclin D1 was also categorized according to stain taken by cells as nuclear alone or combined nuclear and cytoplasmic.

Immunoexpression of Cyclin D1 was seen in 24/40 (60\%) cases with the rest $16(40 \%)$ being negative for Cyclin D1 expression. Holland et al and Bahnssay et al have recorded similar findings with Cyclin D1 expression seen in 58.7\% and $68 \%$ cases respectively whereas Utsunomiya $\mathrm{T}$ and co-workers reported relatively lower (43\%) expression of Cyclin D1 in patients with Colon cancer. ${ }^{10-14}$ The intensity of staining in most of the cases varied from mild (16.7\%) to strong (54.1). The percentage of cells expressing Cyclin D1 varied from $<5 \%$ to $75 \%$. A scoring system was done by adding these two parameters. A scoring system was done on similar lines by Al-Maghrabi $\mathrm{J}$ et $\mathrm{al}^{15}$ and Bahnassy et a ${ }^{11}$

The pattern of staining in the majority of cases (83.3\%) was nuclear while it was both nuclear and cytoplasmic in the rest of the 4 cases $(16.7 \%)$. No case showed a pure cytoplasmic staining pattern. All 4 cases that showed both nuclear and cytoplasmic staining belonged to conventional adenocarcinoma cases. Bahnssay et al had similar findings where Cyclin D1 was predominantly nuclear. Studies done by Fang $\mathrm{YJ}^{16}$ and Mao $\mathrm{Y}^{17}$ showed both nuclear and cytoplasmic staining patterns.

Of the 40 cases studied, most (54\%) of these showed strong intensity/score 3 of staining by Cyclin D1 with mild intensity/score 1 seen in $16.7 \%$ cases. When stratifying cases according to the percentage of cells positive, the maximum number of cases $(50 \%)$ were present in a range of $26-50 \%$ followed by $20.8 \%$ of cases in a range of $51-75 \%$. For statistical purposes, Cyclin D1 immuno-scores were trichotomized as no expression (score 0 ), weak expression (1-4), and strong expression (5-7). Most of the cases (54.2\%) were of weak expression followed by a strong expression (45.8\%). A study done by Al-Maghrabi $\mathrm{J}$ et al showed $77 \%$ of cases had weak expression while $23 \%$ had a strong expression based on a scoring system. ${ }^{15}$

As there was female preponderance in the present study therefore more Cyclin D1 expression (66.7\%) was seen in females in comparison to males $(33.3 \%)$. However, no statistical significance was seen in the expression of Cyclin D1 with the sex of the patient. A study done by AlMaghrabi J et al had resulted in similar lines with Cyclin D1 immunoexpression seen in $52 \%$ females and $44 \%$ of males.

In the present study, it was found that Cyclin D1 was highly positive in CRC patients in the age group ranging between $4^{\text {th }}$ and $5^{\text {th }}$ decade $(77.3 \%)$ of life, and this correlation had high significance with a p-value of 0.033 . Al-Maghrabi J et al showed $55.5 \%$ expression of Cyclin D1 in patients younger than 60 and $44.5 \%$ in $>60$ years old. ${ }^{15}$ It was noted in the present study that Cyclin D1 expression was seen more in tumors of the left side of the colon, notably in the sigmoid and descending colon with $86 \%$ and $50 \%$ expression respectively. This correlation was not statistically significant in agreement with findings by Al-Maghrabi J et al while Jang KY et al found that there was no correlation between Cyclin D1 expression and location of the tumor. ${ }^{15,18}$ Cyclin D1 was positive in $62.5 \%$ of cases with tumor size $>5$ $\mathrm{cm}$ and $53.8 \%$ in cases having lesion size ranging between $2-5 \mathrm{~cm}$, being similar to results reported by Judah et al and Bahnassy et al. ${ }^{15,11}$

In the present study, Cyclin D1 expression was seen in $70 \%$ of cases of conventional adenocarcinoma while only in $30 \%$ cases of mucinous adenocarcinoma. There was a highly significant correlation between Cyclin D1 expression and histological distribution of cases similar to findings reported by Raafat et al. ${ }^{13}$ Cyclin D1 expression was seen in $40 \%$ (2/5) cases of well-differentiated, 75\% (15/20) in moderately differentiated, and $46.7 \%(7 / 15)$ in poorly differentiated cases. Judah et al reported maximum cases $(51.9 \%)$ of Cyclin D1 expression belonged to moderately differentiated adenocarcinoma followed by well-differentiated (37\%) which was in concordance with the present study. ${ }^{15}$ In contrast to the observations in the present study, Bahnassy et al reported almost twice the expression of Cyclin D1 in poorly differentiated conventional adenocarcinoma than moderate and well-differentiated adenocarcinomas.

In 21 cases where lymph nodes were recovered, metastasis was seen in 10 cases and Cyclin D1 was expressed in over half $(60 \%)$ of these cases while in cases with reactive lymph node and Cyclin D1 positivity was slightly more than half (54.5\%). So Cyclin D1 expression was almost equal with the number of lymph nodes that showed metastatic or reactive pathology, hence was found statistically not significant which is similar to work by Kouraklis G et al. ${ }^{19}$

In the present study, $11(64.7 \%)$ of the 17 cases invading serosa showed Cyclin D1 expression while only $1(25 \%)$ of the 4 cases had positivity for Cyclin D1 which had tumor infiltration up to muscle in agreement with findings by Judah et al. ${ }^{15}$

In the present study, Cyclin D1 expression in cases having vascular invasion was seen in half $(50 \%)$ of the cases while in cases where no vascular invasion was seen $63.6 \%$ cases had Cyclin D1 expression. In a study done by Sakarias Wangefjord et al, they found that there was an almost equal expression of Cyclin D1 with presence or absence of vascular invasion, 28.5 and $29.6 \%$ respectively while in a 
study done by Judah et al it was seen that there were more Cyclin D1 expression, $77.8 \%$ cases, where no vascular invasion was seen while in cases having vascular invasion showed $22.4 \%$ positivity. ${ }^{12,15}$

The Crohn's-like reaction is defined as a transmural lymphoid reaction. It was observed in our study that $100 \%$ of the cases showing CLR had Cyclin D1 expression. There was a highly significant correlation between Cyclin D1 expression and CLR shown by a p-value of 0.017 . Similar results were observed with the peritumoral lymphocytic response which is defined as discrete lymphoid reactions surrounding the tumor. As 20/26 cases with PTLR were positive for Cyclin D1 expression and a highly significant correlation was noted between the two with a p-value of 0.003 . Similar findings were observed by Lewis B et al in their cohort study. ${ }^{20}$ Study done by Lewis B et al showed longer survival of patients of CRC showing CLR and PTLR. ${ }^{20}$

\section{CONCLUSIONS}

A statistically significant correlation of Cyclin D1 expression was seen with age group (5th- 6 th decade) and histological type of tumor. No significant correlation of Cyclin D1 expression was seen with lymph node involvement, depth of invasion, vascular invasion, or perineural invasion. A significant statistical correlation was seen with Crohn's s-like reaction and peritumoral lymphocytic response.

\section{Conflict of interest: None}

\section{REFERENCES}

1. Hanahan D, Weinberg RA. Hallmarks of Cancer: 1. Bernard L. Nutrition and colorectal cancer. Cancer. 1992;70:1723-6. $\underline{\text { Crossref }}$

2. Jemal A, Ward EM, Johnson CJ, et al. Annual Report to the Nation on the Status of Cancer, 1975-2014, Featuring Survival. J Natl Cancer Inst. 2017;109: djx030. Crossref

3. Hamilton W, Round A, Sharp D, et al. Clinical features of colorectal cancer before diagnosis: a population-based case-control study. Br J Cancer. 2005;93:399-405. Crossref

4. Parkin DM, Pisani P, Ferlay J. Global cancer statistics. CA Cancer J Clin. 1999;49:33-64. $\underline{\text { Crossref }}$

5. Nakagawa H, Ito H, Hosono S, et al. Changes in trends in colorectal cancer incidence rate by anatomic site between 1978 and 2004 in Japan. Eur J Cancer Prev. 2017 ;26:269-76. $\underline{\text { Crossref }}$

6. Edge SB, Compton CC. The American Joint Committee on Cancer: the 7th edition of the AJCC cancer staging manual and the future of TNM. Ann Surg Oncol. 2010;17:1471-4. Crossref

7. Rex DK, Rahmani EY, Haseman JH, et al. Relative sensitivity of colonoscopy and barium enema for detection of colorectal cancer in clinical practice. Gastroenterology. 1997;112:17-23. Crossref
8. Atkin W, Dadswell E, Wooldrage K, et al. Computed tomographic colonography versus colonoscopy for investigation of patients with symptoms suggestive of colorectal cancer (SIGGAR): a multicentre randomized trial. Lancet. 2013;38:1194-202. Crossref

9. Locker GY, Hamilton S, Harris J, et al. ASCO 2006 update of recommendations for the use of tumor markers in gastrointestinal cancer. J Clin Oncol. 2006;24:5313-27. $\underline{\text { Crossref }}$

10. Holland TA, Elder J, McCloud JM, et al. Subcellular localisation of cyclin D1 protein in colorectal tumours is associated with $\mathrm{p} 21$ (WAF1/ CIP1) expression and correlates with patient survival. Int J Cancer. 2001;95:302-6. $\underline{\text { Crossref }}$

11. Bahnassy AA, Zekri AR, El-Houssini S, et al. Cyclin A and cyclin D1 as significant prognostic markers in colorectal cancer patients. BMC Gastroenterol. 4, 22 (2004). Crossref

12. Wangefjord S, Manjer J, Gaber A, et al. Cyclin D1 expression in colorectal cancer is a favorable prognostic factor in men but not in women in a prospective, population-based cohort study. Biol Sex Differ. 2011;2:10. $\underline{\text { Crossref }}$

13. RaafatAM, Mahmoud TE. Hend FA, et al. Cyclin D1 level, ploidy status, and S-phase fraction in colorectal cancer patients. Egyptian Journal of Pathology. 2015;35:144-9. Crossref

14. Utsunomiya $\mathrm{T}$, Doki $\mathrm{Y}$, Takemoto $\mathrm{H}$, et al. Correlation of beta-catenin and cyclin D1 expression in colon cancers. Oncology. 2001;61:22633. $\underline{\text { Crossref }}$

15. Al-Maghrabi J, Mufti S, Gomaa W, et al. Immunoexpression of cyclin D1 in colorectal carcinomas is not correlated with survival outcome. J Microsc Ultrastruct. 2015;3:62-67. $\underline{\text { Crossref }}$

16. Fang YJ, Lu ZH, Wang GQ, et al. Elevated expressions of MMP7, TROP2, and survivin are associated with survival, disease recurrence, and liver metastasis of colon cancer. Int J Colorectal Dis. 2009;24:875-84. Crossref

17. Mao Y, Li Z, Lou C, et al. Expression of phosphorylated Stat5 predicts expression of cyclin D1 and correlates with poor prognosis of colonic adenocarcinoma. Int J Colorectal Dis. 2011;26:29-35. Crossref

18. Jang KY, Kim YN, Bae JS, et al. Expression of Cyclin D1 Is Associated with $\beta$-Catenin Expression and Correlates with Good Prognosis in Colorectal Adenocarcinoma. Transl Oncol. 2012;5:3708. $\underline{\text { Crossref }}$

19. Kouraklis G, Theocharis S, Vamvakas P, et al. Cyclin D1 and Rb protein expression and their correlation with prognosis in patients with colon cancer. World J Surg Oncol. 2006;4:5. Crossref

20. Lewis $\mathrm{B}, \mathrm{Lin} \mathrm{J}, \mathrm{Wu} \mathrm{X}$, et al. Crohn's disease-like reaction predicts favorable prognosis in colitis-associated colorectal cancer. Inflamm Bowel Dis. 2013;19:2190-8. Crossref 